\title{
The existence and asymptotic estimations of solutions to stochastic pantograph equations with diffusion and Lévy jumps
}

\author{
Wei $\mathrm{Mao}^{1 *}$, Liangjian $\mathrm{Hu}^{2}$, Xuerong $\mathrm{MaO}^{3}$ \\ 1.School of mathematics and information technology, Jiangsu Second Normal University, \\ Nanjing, 210013, P.R.China; \\ 2.Department of Applied Mathematics, Donghua University, Shanghai, \\ 201620,P.R.China; \\ 3.Department of Statistics and Modelling Science, University of Strathclyde, Glasgow, \\ G1 $1 X H, U K$.
}

\begin{abstract}
In this paper, we consider a class of stochastic pantograph differential equations with Lévy jumps (SPDEwLJs). By using the Burkholder-Davis-Gundy inequality and the Kunita's inequality, we prove the existence and uniqueness of solutions to SPDEwLJs whose coefficients satisfying the Lipschitz conditions and the local Lipschitz conditions. Meantime, we establish the p-th exponential estimations and almost surely asymptotic estimations of solutions to SPDEwLJs.
\end{abstract}

Key words: Stochastic pantograph differential equations, Lévy jumps, Existence and uniqueness, Exponential estimations, Almost surely asymptotic estimations.

\section{Introduction}

It is well known that the following differential equation

$$
\begin{aligned}
& x^{\prime}(t)=a x(t)+b x(q t), \quad t \geq 0, q \in(0,1), \\
& x(0)=x_{0}
\end{aligned}
$$

is a linear pantograph equation with unbounded delay and it is a very special delay differential equation. Since $q t<t$, equation (1) is a differential equation with the delay $t-q t$. We note that $q t$ satisfies $q t \rightarrow \infty$ as $t \rightarrow \infty$, but the 
delay $t-q t$ is unbounded. Equation (1) arises in different fields of pure and applied mathematics such as dynamical systems, probability, quantum mechanics and electrodynamics (see $[1,2]$ ) and its generalisations possess a wide range of applications. In $[3,4]$, the authors studied the existence, uniqueness and asymptotic properties of solution to equation (1). Numerical analysis for solution to equation (1) are studied by [5,6]. Meantime, many authors extended the study on equation (1) to the case of non-linear pantograph differential equation and obtained many results about existence, stability and numerical analysis of solution to non-linear pantograph delay equation (see [7-9]).

As differential equation, delay differential equation are often perturbed by the external noise and it is described by stochastic delay differential equation (SDDEs). During the past few decades, qualitative theory of SDDEs have been studied intensively for many authors. One can see [10-20] and the references therein. As a special case of SDDEs, stochastic pantograph differential equations (SPDEs) has been received a great deal of attention. SPDEs can be regarded as a pantograph differential equation perturped by Brownian motion. For example, existence and stability of solution to SPDEs are given in [21-23]. Various efficient computational methods are obtained and their convergence and stability have been studied by many authors [24-29].

It is worth pointing out that the above works only focused on the case of SDDEs driven by a Brownian motion. However, a Brownian motion can not be used to describe the stochastic disturbances in some real systems such as the fluctuations in the financial markets. There is evidence that the dynamics of prices of financial instruments exhibit jumps which cannot be adequately described solely by Brownian motion (see [30]). Also, there are empirical studies, such as [31], which demonstrate the existence of jumps in stock markets and the foreign exchange market. For this case, it is recognized that stochastic differential equations (SDEs) with jump are quite suitable to describe such stochastic abrupt phenomena. For example, SDEs with jump are used to delineate large market fluctuations such as rallies or crashes in financial market [32]. In recent years, SDEs with Poisson processes $N(t)$ have been studied by many authors (see [33-36]). However, if the perturbation is a random process, then the number of the points where jumps occur is random, moreover, the jump values taken at the jump points are also random. In this case, it is not enough to describe the above perturbation by using Poisson processes $N(t)$. It is natural to consider the general jump processes arising from Poisson random measures $N_{\bar{p}}(t, A)$ generated by a Poisson point process 
$\bar{p}(t)$ (see Section 2 for more details). One should note that one important and widely used class of Poisson random measures are the one associated to a Lévy process. By Lévy-Itô decomposition, Lévy processes can be regarded as processes with a Brownian motion and an independent Poisson random measures. In this case, we call that Poisson random measures $N_{\bar{p}}(t, A)$ is a Lévy jump. SDEs driven by Levy jumps have become extremely popular for modeling financial, physical and biological phenomena and many results about such equations have been studied intensively for many authors. For example, Ikeda [37], Applebaum [38] and Rong [39] are devoted to the study of such equations both in theory and their applications; Bass [40] and Albeverio [41] focused on the study about existence and uniqueness of SDEs with Poisson random measure; Oksendal [42] have studied the optimal control, optimal stopping and impulse control for jump diffusion processes; Li [43] discussed the almost sure stability of linear stochastic differential equations with jumps; Applebaum [44] investigated almost sure exponential stability and moment exponential stability of SDEs with Lévy noise; Applebaum [45] showed that perturbed system with Brownian motion and Poisson random measure is almost surely exponentially stable and estimated the corresponding Lyapunov exponents; Zhu [46] studied the asymptotic stability in the p-th moment and almost sure stability for SDEs with Lévy jump.

Motivated by the above works, this paper is concerned with stochastic pantograph differential equations with Lévy jumps (SPDEwLJs)

$$
\begin{aligned}
d x(t) & =f(t, x(t), x(q t)) d t+g(t, x(t), x(q t)) d w_{t}+d Q(t), \quad t \in\left[t_{0}, T\right], \\
Q(t) & =\int_{t_{0}}^{t} \int_{U} h(x(s), x(q s), u) N_{\bar{p}}(d s, d u), \\
x(t) & =\varphi(t), \quad t \in\left[q t_{0}, t_{0}\right] .
\end{aligned}
$$

where $0<q<1$. To the best of our knowledge, there are no literatures concerned with the p-th exponential estimations and almost surely asymptotic estimations of solution to equation (2). On one hand, we prove that equation (2) has a unique solution $x(t)$ in the sense of the $L^{P}$ norm. Our approach is similar to the one in [11]. But we don't use the fixed point Theorem in [22]. Instead, we get the solution of equation (2) via successive approximations. On the other hand, we study the p-th exponential estimations and almost surely asymptotic estimations of solution to equation (2). By the Burkholder-Davis-Gundy inequality, the Kunita's inequality and It $\widehat{o}$ formula, we show that the P-th moment of solution will grow at most expo- 
nentially with exponent $M_{2}$. Moreover, we prove that the p-th exponential estimations implies almost surely asymptotic estimations and give an upper bound for the sample Lyapunov exponent. Meantime, it should be pointed out that the proof for SPDEwLJs is certainly not a straightforward generalization of that for SPDEs without Lévy jumps and some new techniques are developed to cope with the difficulties due to Lévy jumps. Some known results in Mao [11], Fan [22] are generalized to cover a class of more general SPDEwLJs.

The rest of the paper is organized as follows. In Section 2, we introduce some notations and hypotheses concerning equation (2); In Section 3, the existence and uniqueness of solution to equation (2) are investigated; In Section 4 , we prove the pth moment of solution will grow at most exponentially with exponent $M_{2}$ and show that the exponential estimations implies the almost surely asymptotic estimations.

\section{Preliminaries}

Let $\left(\Omega, \mathcal{F},\left\{\mathcal{F}_{t}\right\}_{t \geq 0}, P\right)$ be a complete probability space with a filtration $\left(\mathcal{F}_{t}\right)_{t \geq 0}$ satisfying the usual condition,(i.e., the filtration $\left(\mathcal{F}_{t}\right)$ is continuous on the right and $\left(\mathcal{F}_{0}\right)$ contains all $P$-null sets.) Here $w(t)$ is an m-dimensional Brownian motion defined on the probability space $(\Omega, \mathcal{F}, P)$ adapted to the filtration $\left(\mathcal{F}_{t}\right)_{t \geq 0}$. Let $t \geq 0$ and $D\left([q t, t], R^{n}\right)$ denote the family of functions $\varphi$ from $[q t, t] \rightarrow R^{n}$ that are right-continuous and have limits on the left. $D\left([q t, t], R^{n}\right)$ is equipped with the norm $\|\varphi\|=\sup _{q t \leq \theta \leq t}|\varphi(\theta)|$, where $|$.$| is the$ Euclidean norm in $R^{n}$. If $A$ is a vector or matrix, its transpose is denoted by $A^{\top}$. If $A$ is a matrix, its norm $\|A\|$ is defined by $\|A\|=\sup \{|A x|$ : $|x|=1\}$. For $p \geq 2, \mathcal{L}_{\mathcal{F}_{t}}^{p}\left([q t, t] ; R^{n}\right)$ denote the family of all $\left(\mathcal{F}_{t}\right)$-measurable, $D\left([q t, t], R^{n}\right)$-valued random variables $\varphi=\{\varphi(\theta): q t \leq \theta \leq t\}$ such that $E\|\varphi\|^{p}<\infty$.

Let $\left(R^{n}, \mathcal{B}\left(R^{n}\right)\right)$ be a measurable space and $\pi(d u)$ a $\sigma$ - finite measure on it. Let $\bar{p}=\bar{p}(t), t \geq t_{0}$ be a stationary $\mathcal{F}_{t^{-}}$adapted and $R^{n}$-valued Poisson point process. Then, for $A \in \mathcal{B}\left(R^{n}-\{0\}\right)$, here $0 \notin$ the closure of $A$, we define the Poisson counting measure $N_{\bar{p}}$ associated with $\bar{p}$ by

$$
N_{\bar{p}}\left(\left(t_{0}, t\right] \times A\right):=\#\left\{t_{0}<s \leq t, \bar{p}(s) \in A\right\}=\sum_{t_{0}<s \leq t} I_{A}(\bar{p}(s))
$$


where \# denotes the cardinality of set $\{$.$\} . For simplicity, we denote$

$$
N_{\bar{p}}(t, A):=N_{\bar{p}}\left(\left(t_{0}, t\right] \times A\right) .
$$

From [37], we can obtain that there exists a $\sigma$ - finite measure $\pi$ such that

$$
E\left[N_{\bar{p}}(t, A)\right]=\pi(A)\left(t-t_{0}\right)
$$

and

$$
P\left(N_{\bar{p}}(t, A)=n\right)=\frac{\exp \left[-\pi(A)\left(t-t_{0}\right)\right]\left[\pi(A)\left(t-t_{0}\right)\right]^{n}}{n !} .
$$

This measure $\pi$ is called the Lévy measure. Moreover, by Doob-Meyer's decomposition theorem, there exists a unique $\left\{\mathcal{F}_{t}\right\}$-adapted martingale $\tilde{N}_{\bar{p}}(t, A)$ and a unique $\left\{\mathcal{F}_{t}\right\}$-adapted natural increasing process $\hat{N}_{\bar{p}}(t, A)$ such that

$$
N_{\bar{p}}(t, A)=\tilde{N}_{\bar{p}}(t, A)+\hat{N}_{\bar{p}}(t, A), \quad t>t_{0} .
$$

Here $\tilde{N}_{\bar{p}}(t, A)$ is called the compensated Poisson random measure and

$$
\hat{N}_{\bar{p}}(t, A)=\pi(A)\left(t-t_{0}\right)
$$

is called the compensator. For more details on the Poisson point process and Lévy jumps, see [37, 38, 39].

For $U \in \mathcal{B}\left(R^{n}-\{0\}\right)$, the integral version of equation (2) is given by

$$
\begin{aligned}
x(t) & =\varphi(t)+\int_{t_{0}}^{t} f(s, x(s), x(q s)) d s+\int_{t_{0}}^{t} g(s, x(s), x(q s)) d w_{s}+Q(t), \\
Q(t) & =\int_{t_{0}}^{t} \int_{U} h(x(s), x(q s), u) N_{\bar{p}}(d s, d u)
\end{aligned}
$$

with initial data $\left\{x(t): q t_{0} \leq t \leq t_{0}\right\}=\varphi \in \mathcal{L}_{\mathcal{F}_{t_{0}}}^{p}\left(\left[q t_{0}, t_{0}\right] ; R^{n}\right)$. Here $0<q<1$ and $f:\left[t_{0}, \infty\right] \times R^{n} \times R^{n} \rightarrow R^{n}, g:\left[t_{0}, \infty\right] \times R^{n} \times R^{n} \rightarrow R^{n \times m}$ and $h: R^{n} \times R^{n} \times U \rightarrow R^{n}$ are both Borel-measurable functions. $x(t)$ is a $n$-dimensional state process, $w_{t}$ is a standard $m$-dimensional Brownian motion and $N_{\bar{p}}(d t, d u)$ is the Poisson random measure given by $N_{\bar{p}}(d t, d u)=$ $\tilde{N}_{\bar{p}}(d t, d u)+\pi(d u) d t$, here $\tilde{N}_{\bar{p}}(d t, d u)$ is the compensated Poisson random measure associated to $N_{\bar{p}}$ and $\pi(d u)$ is the Lévy measure associated to $N_{\bar{p}}$. 
Let the following assumptions hold.

(H1) For each $t \in\left[t_{0}, T\right]$ and $p \geq 2$, there exist two positive constants $k_{1}$ and $k_{2}$ such that for all $x_{1}, y_{1}, x_{2}, y_{2} \in R^{n}$,

$$
\begin{aligned}
\left|f\left(t, x_{1}, y_{1}\right)-f\left(t, x_{2}, y_{2}\right)\right|^{2} & \vee\left|g\left(t, x_{1}, y_{1}\right)-g\left(t, x_{2}, y_{2}\right)\right|^{2} \\
& \leq k_{1}\left(\left|x_{1}-x_{2}\right|^{2}+\left|y_{1}-y_{2}\right|^{2}\right)
\end{aligned}
$$

and

$$
\int_{U}\left|h\left(x_{1}, y_{1}, u\right)-h\left(x_{2}, y_{2}, u\right)\right|^{p} \pi(d u) \leq k_{2}\left(\left|x_{1}-x_{2}\right|^{p}+\left|y_{1}-y_{2}\right|^{p}\right) .
$$

(H2) For each $t \in\left[t_{0}, T\right]$ and $p \geq 2$, there exist two positive constants $L_{1}$ and $L_{2}$ such that for all $x, y \in R^{n}$,

$$
|f(t, x, y)|^{2} \vee|g(t, x, y)|^{2} \leq L_{1}\left(1+|x|^{2}+|y|^{2}\right) \text {, }
$$

and

$$
\int_{U}|h(x, y, u)|^{p} \pi(d u) \leq L_{2}\left(1+|x|^{p}+|y|^{p}\right) .
$$

Now we present the definition of solution to equation (3).

Definition 2.1 A right continuous with left limits process $x=\{x(t), t \in$ $\left.\left[q t_{0}, T\right]\right\}\left(t_{0}<T<\infty\right)$ is called a solution of equation (3) with initial data $\varphi(t)$ if

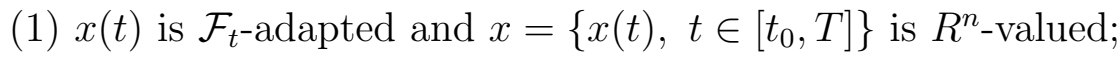

(2) $\int_{q t_{0}}^{T}|x(t)|^{p} d s<\infty$, a.s. for any $p \geq 2$;

(3) $x(t)$ satisfies equation (3).

A solution $x(t)$ is said to be unique if any other solution $y(t)$ is indistinguishable from it, that is,

$$
P\left\{x(t)=y(t), t \in\left[q t_{0}, T\right]\right\}=1 .
$$




\section{The existence and uniqueness theorem}

In this section, we prove the existence and uniqueness of a solution to equation (3).

Define $x^{0}(t)=\varphi\left(t_{0}\right)$, for $t \in\left[t_{0}, T\right]$. For each $n=1,2, \cdots$, set $x^{n}(t)=$ $\varphi(t), t \in\left[q t_{0}, t_{0}\right]$. We introduce the sequence of successive approximations defined as follows:

$$
\begin{aligned}
x^{n}(t)= & \varphi\left(t_{0}\right)+\int_{t_{0}}^{t} f\left(s, x^{n-1}(s), x^{n-1}(q s)\right) d s \\
& +\int_{t_{0}}^{t} g\left(s, x^{n-1}(s), x^{n-1}(q s)\right) d w_{s} \\
& +\int_{t_{0}}^{t} \int_{U} h\left(x^{n-1}(s), x^{n-1}(q s), u\right) N_{\bar{p}}(d s, d u), \quad t \in\left[t_{0}, T\right] .
\end{aligned}
$$

Theorem 3.1 Let $p \geq 2$ and conditions (H1)-(H2) hold, then there exists a unique solution $x(t)$ to equation (3) and the solution $x(t) \in \mathcal{M}^{p}\left(\left[t_{0}, T\right] ; R^{n}\right)$.

To prove Theorem 3.1, we give the following lemmas 3.1 and 3.2.

Lemma 3.1 Under conditions (H1)-(H2), there exists a positive constant $c$ such that

$$
E \sup _{t_{0} \leq t \leq T}\left|x^{n}(t)\right|^{p} \leq c
$$

where $c=\left(c_{6}+c_{7}\left(T-t_{0}\right) E\|\varphi\|^{p}\right) e^{c_{7}\left(T-t_{0}\right)}$.

Proof: For simplicity, we denote

$$
f_{t}^{n}=f\left(t, x^{n}(t), x^{n}(q t)\right), \quad g_{t}^{n}=g\left(t, x^{n}(t), x^{n}(q t)\right), h_{t}^{n}=h\left(x^{n}(t), x^{n}(q t), u\right) .
$$

For every integer $R \geq 1$, define the stopping time

$$
\tau_{R}=T \wedge \inf \left\{t \in\left[t_{0}, T\right]:\left|x^{n-1}(t)\right| \vee\left|x^{n-1}(q t)\right| \geq R\right\} .
$$

Using the basic inequality $|a+b+c+d|^{p} \leq 4^{p-1}\left[|a|^{p}+|b|^{p}+|c|^{p}+|d|^{p}\right]$, it is easy to see from (4) that

$$
E \sup _{t_{0} \leq s \leq t}\left|x^{n}\left(s \wedge \tau_{R}\right)\right|^{p}
$$




$$
\begin{aligned}
\leq & \left.4^{p-1} E|| \varphi\right|^{p}+4^{p-1} E\left(\sup _{t_{0} \leq s \leq t}\left|\int_{t_{0}}^{s} f_{\sigma \wedge \tau_{R}}^{n-1} d \sigma\right|^{p}\right)+4^{p-1} E\left(\sup _{t_{0} \leq s \leq t}\left|\int_{t_{0}}^{s} g_{\sigma \wedge \tau_{R}}^{n-1} d w_{\sigma}\right|^{p}\right) \\
& +4^{p-1} E\left(\sup _{t_{0} \leq s \leq t}\left|\int_{t_{0}}^{s} \int_{U} h_{\sigma \wedge \tau_{R}}^{n-1} N_{\bar{p}}(d \sigma, d u)\right|^{p}\right)
\end{aligned}
$$

Let us estimate the terms introduced above. By the Hölder inequality and (H2), we get

$$
\begin{aligned}
& E\left(\sup _{t_{0} \leq s \leq t}\left|\int_{t_{0}}^{s} f_{\sigma \wedge \tau_{R}}^{n-1} d \sigma\right|^{p}\right) \leq\left(t-t_{0}\right)^{p-1} \int_{t_{0}}^{t} E\left|f_{s \wedge \tau_{R}}^{n-1}\right|^{p} d s \\
\leq & \left(T-t_{0}\right)^{p-1} \int_{t_{0}}^{t} E\left[L_{1}\left(1+\left|x^{n-1}\left(s \wedge \tau_{R}\right)\right|^{2}+\left|x^{n-1}\left(q s \wedge \tau_{R}\right)\right|^{2}\right)\right]^{\frac{p}{2}} d s \\
\leq & \left(T-t_{0}\right)^{p-1} L_{1}^{\frac{p}{2}} 3^{\frac{p}{2}-1} \int_{t_{0}}^{t} E\left(1+\left|x^{n-1}\left(s \wedge \tau_{R}\right)\right|^{p}+\left|x^{n-1}\left(q s \wedge \tau_{R}\right)\right|^{p}\right) d s \\
\leq & c_{1}\left(T-t_{0}\right)+2 c_{1} \int_{t_{0}}^{t} E\left(\sup _{t_{0} \leq \sigma \leq s}\left|x^{n-1}\left(\sigma \wedge \tau_{R}\right)\right|^{p}\right) d s
\end{aligned}
$$

where $c_{1}=\left(T-t_{0}\right)^{p-1} L_{1}^{\frac{p}{2}} 3^{\frac{p}{2}-1}$. Now, using the Burkholder-Davis-Gundy inequality and the Hölder inequality, we have a positive real number $c_{p}$ such that the following inequality holds:

$$
\begin{aligned}
E\left(\sup _{t_{0} \leq s \leq t}\left|\int_{t_{0}}^{s} g_{\sigma \wedge \tau_{R}}^{n-1} d w_{\sigma}\right|^{p}\right) & \leq c_{p} E\left(\int_{t_{0}}^{t}\left|g_{s \wedge \tau_{R}}^{n-1}\right|^{2} d s\right)^{\frac{p}{2}} \\
& \leq c_{p}\left(T-t_{0}\right)^{\frac{p}{2}-1} E \int_{t_{0}}^{t}\left|g_{s \wedge \tau_{R}}^{n-1}\right|^{p} d s .
\end{aligned}
$$

Similar to (7), we obtain

$$
\begin{aligned}
E\left(\sup _{t_{0} \leq s \leq t}\left|\int_{t_{0}}^{s} g_{\sigma \wedge \tau_{R}}^{n-1} d w_{\sigma}\right|^{p}\right) \leq & c_{2}\left(T-t_{0}\right) \\
& +2 c_{2} \int_{t_{0}}^{t} E\left(\sup _{t_{0} \leq \sigma \leq s}\left|x^{n-1}\left(\sigma \wedge \tau_{R}\right)\right|^{p}\right) d s,
\end{aligned}
$$

where $c_{2}=c_{p} L_{1}^{\frac{p}{2}}\left(3\left(T-t_{0}\right)\right)^{\frac{p}{2}-1}$. Next, we estimate the last term of (6). By the definition of $N_{\bar{p}}(d t, d u)=\tilde{N}_{\bar{p}}(d t, d u)+\pi(d u) d t$ and the basic inequality $|a+b|^{p} \leq 2^{p-1}\left(|a|^{p}+|b|^{p}\right)$, we have

$$
E\left(\sup _{t_{0} \leq s \leq t}\left|\int_{t_{0}}^{s} \int_{U} h_{\sigma \wedge \tau_{R}}^{n-1} N_{\bar{p}}(d \sigma, d u)\right|^{p}\right)
$$




$$
\begin{aligned}
= & E\left(\sup _{t_{0} \leq s \leq t}\left|\int_{t_{0}}^{s} \int_{U} h_{\sigma \wedge \tau_{R}}^{n-1} \tilde{N}_{\bar{p}}(d \sigma, d u)+\int_{t_{0}}^{s} \int_{U} h_{\sigma \wedge \tau_{R}}^{n-1} \pi(d u) d \sigma\right|^{p}\right) \\
\leq & 2^{p-1} E\left(\sup _{t_{0} \leq s \leq t}\left|\int_{t_{0}}^{s} \int_{U} h_{\sigma \wedge \tau_{R}}^{n-1} \tilde{N}_{\bar{p}}(d \sigma, d u)\right|^{p}\right) \\
& +2^{p-1} E\left(\sup _{t_{0} \leq s \leq t}\left|\int_{t_{0}}^{s} \int_{U} h_{\sigma \wedge \tau_{R}}^{n-1} \pi(d u) d \sigma\right|^{p}\right) .
\end{aligned}
$$

For the second term of (9), by the Hölder inequality, we have

$$
\begin{aligned}
& E\left(\sup _{t_{0} \leq s \leq t}\left|\int_{t_{0}}^{s} \int_{U} h_{\sigma \wedge \tau_{R}}^{n-1} \pi(d u) d \sigma\right|^{p}\right) \\
\leq & E \sup _{t_{0} \leq s \leq t}\left\{\left[\int_{t_{0}}^{s} d \sigma\right]^{p-1} \int_{t_{0}}^{s}\left|\int_{U} h_{\sigma \wedge \tau_{R}}^{n-1} \pi(d u)\right|^{p} d \sigma\right\} \\
\leq & \left(t-t_{0}\right)^{p-1} E \int_{t_{0}}^{t}\left|\int_{U} h_{\sigma \wedge \tau_{R}}^{n-1} \pi(d u)\right|^{p} d s \\
\leq & \left(T-t_{0}\right)^{p-1} E \int_{t_{0}}^{t}\left\{\left[\int_{U} \pi(d u)\right]^{\frac{p}{2}}\left[\int_{U}\left|h_{s \wedge \tau_{R}}^{n-1}\right|^{2} \pi(d u)\right]^{\frac{p}{2}}\right\} d s \\
\leq & \left(T-t_{0}\right)^{p-1}[\pi(U)]^{\frac{p}{2}} E \int_{t_{0}}^{t}\left[L_{2}\left(1+\left|x^{n-1}\left(s \wedge \tau_{R}\right)\right|^{2}+\left|x^{n-1}\left(q s \wedge \tau_{R}\right)\right|^{2}\right)\right]^{\frac{p}{2}} d s \\
\leq & c_{3}\left(T-t_{0}\right)+2 c_{3} \int_{t_{0}}^{t} E\left(\sup _{t_{0} \leq \sigma \leq s}\left|x^{n-1}\left(\sigma \wedge \tau_{R}\right)\right|^{p}\right) d s,
\end{aligned}
$$

where $c_{3}=\left(T-t_{0}\right)^{p-1} L_{2}^{\frac{p}{2}}(\pi(U))^{\frac{p}{2}} 3^{\frac{p}{2}-1}$. Now let us estimate the first term in (9). By the Kunita's first inequality (see Applebaum [38] and Kunita [47]), we have a positive real number $D_{p}$ such that

$$
\begin{aligned}
& E \sup _{t_{0} \leq s \leq t}\left|\int_{t_{0}}^{s} \int_{U} h_{\sigma \wedge \tau_{R}}^{n-1} \tilde{N}_{\bar{p}}(d \sigma, d u)\right|^{p} \\
\leq & D_{p}\left\{E\left[\int_{t_{0}}^{t} \int_{U}\left|h_{s \wedge \tau_{R}}^{n-1}\right|^{2} \pi(d u) d s\right]^{\frac{p}{2}}+E\left[\int_{t_{0}}^{t} \int_{U}\left|h_{s \wedge \tau_{R}}^{n-1}\right|^{p} \pi(d u) d s\right]\right\} .
\end{aligned}
$$

In the same way as (10) was done, it follows from (11) that

$$
\begin{aligned}
& E \sup _{t_{0} \leq s \leq t}\left|\int_{t_{0}}^{s} \int_{U} h_{\sigma \wedge \tau_{R}}^{n-1} \tilde{N}_{\bar{p}}(d \sigma, d u)\right|^{p} \\
\leq & D_{p}\left[3^{\frac{p}{2}-1} L_{2}^{\frac{p}{2}}\left(T-t_{0}\right)^{\frac{p}{2}-1}+L_{2}\right] E \int_{t_{0}}^{t}\left(1+\left|x^{n-1}\left(s \wedge \tau_{R}\right)\right|^{p}\right.
\end{aligned}
$$




$$
\begin{aligned}
& \left.+\left|x^{n-1}\left(q s \wedge \tau_{R}\right)\right|^{p}\right) d s \\
\leq & c_{4}\left(T-t_{0}\right)+2 c_{4} \int_{t_{0}}^{t} E\left(\sup _{t_{0} \leq \sigma \leq s}\left|x^{n-1}\left(\sigma \wedge \tau_{R}\right)\right|^{p}\right) d s,
\end{aligned}
$$

where $c_{4}=D_{p}\left[3^{\frac{p}{2}-1} L_{2}^{\frac{p}{2}}\left(T-t_{0}\right)^{\frac{p}{2}-1}+L_{2}\right]$. Inserting (10) and (12) into (9), we obtain

$$
\begin{aligned}
& E\left(\sup _{t_{0} \leq s \leq t}\left|\int_{t_{0}}^{s} \int_{U} h_{\sigma \wedge \tau_{R}}^{n-1} N_{\bar{p}}(d \sigma, d u)\right|^{p}\right) \\
\leq & c_{5}\left(T-t_{0}\right)+2 c_{5} \int_{t_{0}}^{t} E\left(\sup _{t_{0} \leq \sigma \leq s}\left|x^{n-1}\left(\sigma \wedge \tau_{R}\right)\right|^{p}\right) d s,
\end{aligned}
$$

where $c_{5}=2^{p-1}\left(c_{3}+c_{4}\right)$. Combing (7), (8) and (13) together, we have

$$
E \sup _{t_{0} \leq s \leq t}\left|x^{n}\left(s \wedge \tau_{R}\right)\right|^{p} \leq c_{6}+c_{7} \int_{t_{0}}^{t} E\left(\sup _{t_{0} \leq \sigma \leq s}\left|x^{n-1}\left(\sigma \wedge \tau_{R}\right)\right|^{p}\right) d s
$$

where $c_{6}=4^{p-1}\left[E\|\varphi\|^{p}+\left(c_{1}+c_{2}+c_{5}\right)\left(T-t_{0}\right)\right], c_{7}=24^{p-1}\left(c_{1}+c_{2}+c_{5}\right)$. For any $r \geq 1$, it follows from (14) that

$$
\begin{aligned}
& \max _{1 \leq n \leq r} E\left(\sup _{t_{0} \leq s \leq t}\left|x^{n}\left(s \wedge \tau_{R}\right)\right|^{p}\right) \\
\leq & c_{6}+c_{7} \int_{t_{0}}^{t} \max _{1 \leq n \leq r} E \sup _{t_{0} \leq \sigma \leq s}\left|x^{n-1}\left(\sigma \wedge \tau_{R}\right)\right|^{p} d s \\
\leq & c_{6}+c_{7} \int_{t_{0}}^{t}\left(E \|\left.\varphi\right|^{p}+\max _{1 \leq n \leq r} E \sup _{t_{0} \leq \sigma \leq s}\left|x^{n}\left(\sigma \wedge \tau_{R}\right)\right|^{p}\right) d s .
\end{aligned}
$$

From the Gronwall inequality, we derive that

$$
\max _{1 \leq n \leq r} E\left(\sup _{t_{0} \leq s \leq t}\left|x^{n}\left(s \wedge \tau_{R}\right)\right|^{p}\right) \leq\left[c_{6}+c_{7}\left(T-t_{0}\right) E\|\varphi\|^{p}\right] e^{c_{7}\left(T-t_{0}\right)} .
$$

Since $r$ is arbitrary, we must have

$$
E\left(\sup _{t_{0} \leq s \leq t}\left|x^{n}\left(s \wedge \tau_{R}\right)\right|^{p}\right) \leq\left(c_{6}+c_{7}\left(T-t_{0}\right) E\|\varphi\|^{p}\right) e^{c_{7}\left(T-t_{0}\right)} .
$$

Letting $R \rightarrow \infty$, we then obtain

$$
E\left(\sup _{t_{0} \leq s \leq t}\left|x^{n}(s)\right|^{p}\right) \leq\left(c_{6}+c_{7}\left(T-t_{0}\right) E\|\varphi\|^{p}\right) e^{c_{7}\left(T-t_{0}\right)}
$$


which shows that the desired result holds with $c=\left(c_{6}+c_{7}\left(T-t_{0}\right) E\|\varphi\|^{p}\right) e^{c_{7}\left(T-t_{0}\right)}$.

Lemma 3.2 Let conditions (H1),(H2) hold, then $\left\{x^{n}(t)\right\}_{n \geq 1}$ defined by (4) is a Cauchy sequence in $D\left(\left[t_{0}, T\right], R^{n}\right)$.

Proof: For $n \geq 1$ and $t \in\left[t_{0}, T\right]$, it follows from (4) that $x^{n+1}(t)-x^{n}(t)=\int_{t_{0}}^{t} f_{s}^{n, n-1} d s+\int_{t_{0}}^{t} g_{s}^{n, n-1} d w_{s}+\int_{t_{0}}^{t} \int_{U} h_{s, u}^{n, n-1} N_{\bar{p}}(d s, d u)$, where

$$
\begin{aligned}
& f_{s}^{n, n-1}=f\left(s, x^{n}(s), x^{n}(q s)\right)-f\left(s, x^{n-1}(s), x^{n-1}(q s)\right) \\
& g_{s}^{n, n-1}=g\left(s, x^{n}(s), x^{n}(q s)\right)-g\left(s, x^{n-1}(s), x^{n-1}(q s)\right) \\
& h_{s, u}^{n, n-1}=h\left(x^{n}(s), x^{n}(q s), u\right)-h\left(x^{n-1}(s), x^{n-1}(q s), u\right) .
\end{aligned}
$$

Thus taking expectation on $\left|x^{n+1}(t)-x^{n}(t)\right|^{p}$, we have

$$
\begin{aligned}
& E\left(\sup _{t_{0} \leq s \leq t}\left|x^{n+1}(s)-x^{n}(s)\right|^{p}\right) \\
\leq & 3^{p-1}\left[E\left(\sup _{t_{0} \leq s \leq t}\left|\int_{0}^{s} f_{\sigma}^{n, n-1} d \sigma\right|^{p}\right)+E\left(\sup _{t_{0} \leq s \leq t}\left|\int_{t_{0}}^{t} g_{\sigma}^{n, n-1} d w_{\sigma}\right|^{p}\right)\right. \\
& \left.+E\left(\sup _{t_{0} \leq s \leq t}\left|\int_{t_{0}}^{t} \int_{U} h_{\sigma, u}^{n, n-1} N_{\bar{p}}(d \sigma, d u)\right|^{p}\right)\right] .
\end{aligned}
$$

By the Hölder inequality and (H1), we obtain that

$$
\begin{aligned}
& E\left(\sup _{t_{0} \leq s \leq t}\left|\int_{t_{0}}^{s} f_{\sigma}^{n, n-1} d \sigma\right|^{p}\right) \\
\leq & \left(t-t_{0}\right)^{p-1} E \int_{t_{0}}^{t}\left|f_{s}^{n, n-1}\right|^{p} d s \\
\leq & \left(T-t_{0}\right)^{p-1} k_{1}^{\frac{p}{2}} 2^{\frac{p}{2}-1} E \int_{t_{0}}^{t}\left[\left|x^{n}(s)-x^{n-1}(s)\right|^{p}+\left|x^{n}(q s)-x^{n-1}(q s)\right|^{p}\right] d s \\
\leq & \left(T-t_{0}\right)^{p-1} k_{1}^{\frac{p}{2}} 2^{\frac{p}{2}} \int_{t_{0}}^{t} E \sup _{0 \leq \sigma \leq s}\left|x^{n}(\sigma)-x^{n-1}(\sigma)\right|^{p} d s .
\end{aligned}
$$

Now, using the Burkholder-Davis-Gundy inequality and Hölder inequality, we get

$$
E\left(\sup _{t_{0} \leq s \leq t}\left|\int_{t_{0}}^{s} g_{\sigma}^{n, n-1} d w_{\sigma}\right|^{p}\right)
$$




$$
\begin{aligned}
& \leq c_{p} E\left(\int_{t_{0}}^{t}\left|g_{s}^{n, n-1}\right|^{2} d s\right)^{\frac{p}{2}} \leq c_{p}\left(t-t_{0}\right)^{\frac{p}{2}-1} E \int_{t_{0}}^{t}\left|g_{s}^{n, n-1}\right|^{p} d s \\
& \leq c_{p}\left(T-t_{0}\right)^{\frac{p}{2}-1} k_{1}^{\frac{p}{2}} 2^{\frac{p}{2}} \int_{t_{0}}^{t} E \sup _{t_{0} \leq \sigma \leq s}\left|x^{n}(\sigma)-x^{n-1}(\sigma)\right|^{p} d s .
\end{aligned}
$$

Similar to (9), by the Kunita's first inequality, Hölder inequality, we have

$$
\begin{aligned}
& E\left(\sup _{t_{0} \leq s \leq t}\left|\int_{t_{0}}^{s} \int_{U} h_{\sigma, u}^{n, n-1} N_{\bar{p}}(d \sigma, d u)\right|^{p}\right) \\
\leq & E\left(\sup _{t_{0} \leq s \leq t}\left|\int_{t_{0}}^{s} \int_{U} h_{\sigma, u}^{n, n-1} \tilde{N}_{\bar{p}}(d \sigma, d u)+\int_{t_{0}}^{s} \int_{U} h_{\sigma, u}^{n, n-1} \pi(d u) d \sigma\right|^{p}\right) \\
\leq & c_{8} E \sup _{t_{0} \leq \sigma \leq s}\left|x^{n}(\sigma)-x^{n-1}(\sigma)\right|^{p} d s
\end{aligned}
$$

where $c_{8}=2^{\frac{3 p}{2}-1} k_{2}^{\frac{p}{2}}\left[D_{p}\left(T-t_{0}\right)^{\frac{p}{2}-1}+(\pi(U))^{\frac{p}{2}}\left(T-t_{0}\right)^{p-1}\right]+2^{p} D_{p} k_{2}$. Inserting (16)-(18) into (15), it follows that

$$
E\left(\sup _{t_{0} \leq s \leq t}\left|x^{n+1}(s)-x^{n}(s)\right|^{p}\right) \leq c_{9} \int_{t_{0}}^{t} E\left(\sup _{t_{0} \leq \sigma \leq s}\left|x^{n}(\sigma)-x^{n-1}(\sigma)\right|^{p}\right) d s,
$$

where $c_{9}=3^{p-1} k_{1}^{\frac{p}{2}} 2^{\frac{p}{2}}\left[\left(T-t_{0}\right)^{\frac{p}{2}-1} c_{p}+\left(T-t_{0}\right)^{p-1}\right]+3^{p-1} c_{8}$. Setting $\varphi_{n}(t)=$ $E \sup _{t_{0} \leq s \leq t}\left|x^{n+1}(s)-x^{n}(s)\right|^{p}$, we have

$$
\begin{aligned}
\varphi_{n}(t) & \leq c_{9} \int_{t_{0}}^{t} \varphi_{n-1}\left(s_{1}\right) d s_{1} \leq c_{9}^{2} \int_{0}^{t} d s_{1} \int_{t_{0}}^{s_{1}} \varphi_{n-2}\left(s_{2}\right) d s_{2} \\
& \leq \cdots \\
& \leq c_{9}^{n} \int_{t_{0}}^{t} d s_{1} \int_{t_{0}}^{s_{1}} d s_{2} \cdots \int_{t_{0}}^{s_{n-1}} \varphi_{0}\left(s_{n}\right) d s_{n} .
\end{aligned}
$$

Using the Burkholder-Davis-Gundy inequality inequality, Hölder inequality and (H2), we have

$$
\varphi_{0}(t)=E \sup _{t_{0} \leq s \leq t}\left|x^{1}(s)-x^{0}(s)\right|^{p} \leq M .
$$

Substituting (21) into (20) and integrating the right hand side, we obtain that

$$
E\left(\sup _{t_{0} \leq s \leq t}\left|x^{n+1}(s)-x^{n}(s)\right|^{p}\right) \leq \frac{M\left(c_{9}\left(t-t_{0}\right)\right)^{n}}{n !} .
$$


For (22), taking $t=T$,

$$
E\left(\sup _{t_{0} \leq t \leq T}\left|x^{n+1}(t)-x^{n}(t)\right|^{p}\right) \leq \frac{M\left(c_{9}\left(T-t_{0}\right)\right)^{n}}{n !} .
$$

Then using the Chebyshev inequality, one gets

$$
\begin{aligned}
& P\left(\sup _{t_{0} \leq t \leq T}\left|x^{n+1}(t)-x^{n}(t)\right|>\frac{1}{2^{n}}\right) \\
\leq & P\left(\left|\int_{t_{0}}^{T} f_{s}^{n, n-1} d s\right|>\frac{1}{3} \frac{1}{2^{n}}\right)+P\left(\sup _{t_{0} \leq t \leq T}\left|\int_{t_{0}}^{t} g_{s}^{n, n-1} d w_{s}\right|>\frac{1}{3} \frac{1}{2^{n}}\right) \\
& +P\left(\sup _{t_{0} \leq t \leq T}\left|\int_{t_{0}}^{t} \int_{U} h_{s, u}^{n, n-1} N_{\bar{p}}(d s, d u)\right|>\frac{1}{3} \frac{1}{2^{n}}\right) \\
\leq & \left.3^{p} 2^{n p}\left(t-t_{0}\right)^{p-1} E \int_{t_{0}}^{T}\left|f_{s}^{n, n-1}\right|^{p} d s+3^{p} 2^{n p} E \sup _{t_{0} \leq t \leq T} \mid \int_{t_{0}}^{t} g_{s}^{n, n-1}\right]\left.d w_{s}\right|^{p} \\
& +3^{p} 2^{n p} E \sup _{t_{0} \leq t \leq T}\left|\int_{t_{0}}^{t} \int_{U} h_{s, u}^{n, n-1} N_{\bar{p}}(d s, d u)\right|^{p} .
\end{aligned}
$$

By a straightforward computation, there exists a positive constant $C$ such that

$$
\begin{aligned}
& P\left(\sup _{t_{0} \leq t \leq T}\left|x^{n+1}(t)-x^{n}(t)\right|>\frac{1}{2^{n}}\right) \\
\leq & C \int_{t_{0}}^{T} E \sup _{t_{0} \leq s \leq t}\left|x^{n}(s)-x^{n-1}(s)\right|^{p} d t \leq \frac{C M\left(c_{9}\left(T-t_{0}\right)\right)^{n}}{n !} .
\end{aligned}
$$

For any $t \in\left[t_{0}, T\right],(24)$ implies that

$$
\Sigma_{n=0}^{\infty} P\left(\sup _{t_{0} \leq t \leq T}\left|x^{n+1}(t)-x^{n}(t)\right|>\frac{1}{2^{n}}\right)<\infty .
$$

By the Borel-Cantelli lemma, we have

$$
P\left(\limsup _{n \rightarrow \infty} \sup _{t_{0} \leq t \leq T}\left|x^{n+1}(t)-x^{n}(t)\right| \leq \frac{1}{2^{n}}\right)=1 .
$$

That is, there exists a set $\Omega \in \mathcal{F}$ with $P(\Omega)=1$ and a positive integer $n_{0}=n_{0}(\omega)$ such that for almost all $\omega \in \Omega$, we have

$$
\sup _{t_{0} \leq t \leq T}\left|x^{n+1}(t)-x^{n}(t)\right| \leq \frac{1}{2^{n}} \text { whenever } n \geq n_{0}(\omega) \text {. }
$$


This implies $\left\{x^{n}(t)\right\}_{n=1,2 \ldots}$ is a Cauchy sequence on $\left[t_{0}, T\right]$ under sup |.|. However, the space $D\left(\left[t_{0}, T\right], R^{n}\right)$ is not a complete space under sup |.| and we cannot get the limit of the sequence $\left\{x^{n}(t)\right\}_{n \geq 1}$. So, we need to introduce a metric to make the space $D\left(\left[t_{0}, T\right], R^{n}\right)$ complete. For any $x, y \in D\left(\left[t_{0}, T\right], R^{n}\right)$, Billing [48] gives the following metric

$$
d(x, y)=\inf _{\lambda \in \Lambda}\left\{\sup _{t_{0} \leq t \leq T}\left|x_{t}-y_{\lambda(t)}\right|+\sup _{t_{0} \leq s \leq t \leq T}\left|\log \frac{\lambda(t)-\lambda(s)}{t-s}\right|\right\},
$$

where $\Lambda=\left\{\lambda=\lambda(t): \lambda\right.$ is strictly increasing, continuous on $t \in\left[t_{0}, T\right]$, such that $\left.\lambda\left(t_{0}\right)=t_{0}, \lambda(T)=T\right\}$. Thus, we have that $\left(D\left(\left[t_{0}, T\right]\right), R^{n}\right)$ is a complete metric space. Taking $\lambda(t)=t$, we can see $\left\{x^{n}(t)\right\}_{n \geq 1}$ is a cauchy sequence under $d(.,$.$) . So the proof is complete.$

Proof of Theorem 3.1 Uniqueness. Let $x(t)$ and $y(t)$ be two solutions of equation (3). Then, by the Burkholder-Davis-Gundy inequality, the Kunita's first inequality and Hölder inequality, we have

$$
E \sup _{t_{0} \leq s \leq t}|x(s)-y(s)|^{p} \leq C \int_{t_{0}}^{t} E \sup _{t_{0} \leq u \leq s}|x(u)-y(u)|^{p} d s .
$$

Therefore, the Gronwall inequality implies,

$$
E \sup _{t_{0} \leq s \leq t}|x(s)-y(s)|^{p}=0, \quad t \in\left[t_{0}, T\right] .
$$

The above expression means that $x(t)=y(t)$ for all $t \in\left[t_{0}, T\right]$. Therefore, for all $t \in\left[t_{0}, T\right], x(t)=y(t)$ a.s.

Existence. We derive from Lemma 3.2 that $\left\{x^{n}(t)\right\}_{n=1,2 \ldots}$ is a Cauchy sequence in $D\left(\left[t_{0}, T\right], R^{n}\right)$. Hence there exists a unique $x(t) \in D\left(\left[t_{0}, T\right], R^{n}\right)$ such that $d\left(x^{n}(),. x().\right) \rightarrow 0$ as $n \rightarrow \infty$. For all $t \in\left[t_{0}, T\right]$, taking limits on both sides of (4) and letting $n \rightarrow \infty$, we can show that $x(t)$ is a solution of equation (3). On the other hand, similar to the proof of lemma 3.1, we can easily obtain that

$$
E|x(t)|^{p} \leq C, \text { for all } t_{0} \leq t \leq T
$$

where $C$ is a constant. Therefore,

$$
E \int_{t_{0}}^{T}|x(t)|^{p} d t \leq C\left(T-t_{0}\right)<\infty .
$$


That is $x(t) \in \mathcal{M}^{p}\left(\left[t_{0}, T\right] ; R^{n}\right)$. So the proof of Theorem 3.1 is complete.

Next, we relax the Lipschitz condition (H1) and replace them by the following local Lipschitz condition.

(H3) For each $t \in\left[t_{0}, T\right]$ and $p \geq 2$, there exists a positive constant $k_{n}$ such that

$\left|f\left(t, x_{1}, y_{1}\right)-f\left(t, x_{2}, y_{2}\right)\right|^{2} \vee\left|g\left(t, x_{1}, y_{1}\right)-g\left(t, x_{2}, y_{2}\right)\right|^{2} \leq k_{n}\left(\left|x_{1}-x_{2}\right|^{2}+\left|y_{1}-y_{2}\right|^{2}\right)$,

and

$$
\int_{U}\left|h\left(x_{1}, y_{1}, u\right)-h\left(x_{2}, y_{2}, u\right)\right|^{p} \pi(d u) \leq k_{n}\left(\left|x_{1}-x_{2}\right|^{p}+\left|y_{1}-y_{2}\right|^{p}\right) .
$$

for all $x_{1}, y_{1}, x_{2}, y_{2} \in R^{n}$ with $\left|x_{1}\right| \vee\left|y_{1}\right| \vee\left|x_{2}\right| \vee\left|y_{2}\right| \leq n$.

Then Theorem 3.1 can be generalized as Theorem 3.2.

Theorem 3.2 Let conditions (H2), (H3) hold, then equation (3) has a unique solution $x(t)$ on $\left[t_{0}, T\right]$.

Proof: For each $n \geq 1$, define the truncation function

$$
f_{n}(t, x, y)= \begin{cases}f(t, x, y), & \text { if } \quad|x| \vee|y| \leq n, \\ f\left(t, \frac{n x}{|x|}, \frac{n y}{|y|}\right), & \text { if }|x| \wedge|y|>n, \\ f\left(t, x, \frac{n y}{|y|}\right), & \text { if }|x| \leq n \text { and }|y|>n, \\ f\left(t, \frac{n x}{|x|}, y\right), & \text { if }|x|>n \text { and }|y| \leq n,\end{cases}
$$

and $g_{n}(t, x, y), h_{n}(x, y, u)$ similarly. Then $f_{n}, g_{n}, h_{n}$ satisfy conditions (H1) and (H2). Let $f_{n}(t)=f_{n}\left(t, x_{n}(t), x_{n}(q t)\right), g_{n}(t)=g_{n}\left(t, x_{n}(t), x_{n}(q t)\right), h_{n}(t, u)=$ $h_{n}\left(x_{n}(t), x_{n}(q t), u\right)$. By Theorem 3.1, we have that equation

$$
x_{n}(t)=\varphi(t)+\int_{t_{0}}^{t} f_{n}(s) d s+\int_{t_{0}}^{t} g_{n}(s) d w_{s}+\int_{t_{0}}^{t} \int_{U} h_{n}(s, u) N_{\bar{p}}(d s, d u)(27)
$$

has a unique solution $x_{n}(t)$, moreover, $x_{n}(t) \in \mathcal{M}^{p}\left(\left[t_{0}, T\right] ; R^{n}\right)$. Of course, $x_{n+1}(t)$ is the unique solution of equation

$x_{n+1}(t)=\varphi(t)+\int_{t_{0}}^{t} f_{n+1}(s) d s+\int_{t_{0}}^{t} g_{n+1}(s) d w_{s}+\int_{t_{0}}^{t} \int_{U} h_{n+1}(s, u) N_{\bar{p}}(d s, d u)$. 
and $x_{n+1}(t) \in \mathcal{M}^{p}\left(\left[t_{0}, T\right] ; R^{n}\right)$. For any fixed $n \geq 1$, define the stopping time

$$
\tau_{n}=T \wedge \inf \left\{t \in\left[t_{0}, T\right]:\left|x_{n}(t)\right| \vee\left|x_{n}(q t)\right| \geq n\right\} .
$$

Taking the expectation on $r_{n}(t)=\left|x_{n+1}(t)-x_{n}(t)\right|^{p}$, we have

$$
\begin{aligned}
E \sup _{t_{0} \leq s \leq t}\left|r_{n}(s)\right|^{p} \leq & \left.3^{p-1}\left[E \sup _{t_{0} \leq s \leq t} \mid \int_{t_{0}}^{s}\left[f_{n+1}(\sigma)\right)-f_{n}(\sigma)\right] d \sigma\right|^{p} \\
& +E \sup _{t_{0} \leq s \leq t}\left|\int_{t_{0}}^{s}\left[g_{n+1}(\sigma)-g_{n}(\sigma)\right] d w_{\sigma}\right|^{p} \\
& \left.+E \sup _{t_{0} \leq s \leq t}\left|\int_{t_{0}}^{s} \int_{U}\left[h_{n+1}(\sigma, u)-h_{n}(\sigma, u)\right] N_{\bar{p}}(d \sigma, d u)\right|^{p}\right] \\
= & 3^{p-1}\left(\mathcal{I}_{1}+\mathcal{I}_{2}+\mathcal{I}_{3}\right)
\end{aligned}
$$

By the Hölder inequality and rearranging the terms on the right-hand side by plus-and minus technique, we get

$$
\begin{aligned}
\mathcal{I}_{1} \leq & \left(t-t_{0}\right)^{p-1} E \int_{t_{0}}^{t}\left|f_{n+1}(s)-f_{n}(s)\right|^{p} d s \\
\leq & \left(2\left(t-t_{0}\right)\right)^{p-1} E \int_{t_{0}}^{t}\left[\left|f_{n+1}(s)-f_{n+1}\left(s, x_{n}(s), x_{n}(q s)\right)\right|^{p}\right. \\
& \left.+\left|f_{n+1}\left(s, x_{n}(s), x_{n}(q s)\right)-f_{n}(s)\right|^{p}\right] d s .
\end{aligned}
$$

Using the Burkholder-Davis-Gundy inequality inequality, the Kunita's first inequality and the Hölder inequality again, it follows that

$$
\begin{aligned}
\mathcal{I}_{2} \leq & c_{p} E\left(\int_{t_{0}}^{t}\left|g_{n+1}(s)-g_{n}(s)\right|^{2} d s\right)^{\frac{p}{2}} \\
\leq & c_{p}\left(t-t_{0}\right)^{\frac{p}{2}-1} E \int_{t_{0}}^{t}\left|g_{n+1}(s)-g_{n}(s)\right|^{p} d s \\
\leq & c_{p}\left(t-t_{0}\right)^{\frac{p}{2}-1} 2^{p-1} E \int_{t_{0}}^{t}\left[\left|g_{n+1}(s)-g_{n+1}\left(s, x_{n}(s), x_{n}(q s)\right)\right|^{p}\right. \\
& \left.+\left|g_{n+1}\left(s, x_{n}(s), x_{n}(q s)\right)-g_{n}(s)\right|^{p}\right] d s .
\end{aligned}
$$

and

$\mathcal{I}_{3} \leq\left(2\left(T-t_{0}\right)\right)^{p-1}[\pi(U)]^{\frac{p}{2}} E \int_{t_{0}}^{t}\left[\int_{U}\left|h_{n+1}(s, u)-h_{n}(s, u)\right|^{2} \pi(d u)\right]^{\frac{p}{2}} d s$ 


$$
\begin{aligned}
& +2^{p-1} D_{p}\left\{E\left[\int_{t_{0}}^{t} \int_{U}\left|h_{n+1}(s, u)-h_{n}(s, u)\right|^{2} \pi(d u) d s\right]^{\frac{p}{2}}\right. \\
& \left.+E\left[\int_{t_{0}}^{t} \int_{U}\left|h_{n+1}(s, u)-h_{n}(s, u)\right|^{p} \pi(d u) d s\right]\right\} \\
\leq & \left(2\left(T-t_{0}\right)\right)^{p-1}[\pi(U)]^{\frac{p}{2}} E \int_{t_{0}}^{t}\left[\int _ { U } \left(2\left|h_{n+1}(s, u)-h_{n+1}\left(x_{n}(s), x_{n}(q s), u\right)\right|^{2}\right.\right. \\
& \left.\left.+2\left|h_{n+1}\left(x_{n}(s), x_{n}(q s), u\right)-h_{n}(s, u)\right|^{2}\right) \pi(d u)\right]^{\frac{p}{2}} d s \\
& +2^{p-1} D_{p} E\left[\int _ { t _ { 0 } } ^ { t } \int _ { U } \left(2\left|h_{n+1}(s, u)-h_{n+1}\left(x_{n}(s), x_{n}(q s), u\right)\right|^{2}\right.\right. \\
& \left.\left.+2\left|h_{n+1}\left(x_{n}(s), x_{n}(q s), u\right)-h_{n}(s, u)\right|^{2}\right) \pi(d u) d s\right]^{\frac{p}{2}} \\
& +2^{2 p-2} D_{p} E \int_{t_{0}}^{t} \int_{U}\left(\left|h_{n+1}(s, u)-h_{n+1}\left(x_{n}(s), x_{n}(q s), u\right)\right|^{p}\right. \\
& \left.+\left|h_{n+1}\left(x_{n}(s), x_{n}(q s), u\right)-h_{n}(s, u)\right|^{p}\right) \pi(d u) d s .
\end{aligned}
$$

Combing (29)-(31) together, it follows that

$$
\begin{aligned}
& E \sup _{t_{0} \leq s \leq t}\left|r_{n}(s)\right|^{p} \\
\leq & \left(6\left(T-t_{0}\right)\right)^{p-1} E \int_{t_{0}}^{t}\left[\left|f_{n+1}(s)-f_{n+1}\left(s, x_{n}(s), x_{n}(q s)\right)\right|^{p}\right. \\
& \left.+\left|f_{n+1}\left(s, x_{n}(s), x_{n}(q s)\right)-f_{n}(s)\right|^{p}\right] d s \\
& +6^{p-1} c_{p}\left(T-t_{0}\right)^{\frac{p}{2}-1} E \int_{t_{0}}^{t}\left[\left|g_{n+1}(s)-g_{n+1}\left(s, x_{n}(s), x_{n}(q s)\right)\right|^{p}\right. \\
& \left.+\left|g_{n+1}\left(s, x_{n}(s), x_{n}(q s)\right)-g_{n}(s)\right|^{p}\right] d s \\
& +\left(6\left(T-t_{0}\right)\right)^{p-1}[\pi(U)]^{\frac{p}{2}} E \int_{t_{0}}^{t}\left[\int _ { U } \left(2\left|h_{n+1}(s, u)-h_{n+1}\left(x_{n}(s), x_{n}(q s), u\right)\right|^{2}\right.\right. \\
& \left.\left.+2\left|h_{n+1}\left(x_{n}(s), x_{n}(q s), u\right)-h_{n}(s, u)\right|^{2}\right) \pi(d u)\right]^{\frac{p}{2}} d s \\
& +6^{p-1} D_{p} E\left[\int _ { t _ { 0 } } ^ { t } \int _ { U } \left(2\left|h_{n+1}(s, u)-h_{n+1}\left(x_{n}(s), x_{n}(q s), u\right)\right|^{2}\right.\right. \\
+ & \left.\left.2\left|h_{n+1}\left(x_{n}(s), x_{n}(q s), u\right)-h_{n}(s, u)\right|^{2}\right) \pi(d u) d s\right]^{\frac{p}{2}} \\
+ & 12^{p-1} D_{p} E \int_{t_{0}}^{t} \int_{U}\left(\left|h_{n+1}(s, u)-h_{n+1}\left(x_{n}(s), x_{n}(q s), u\right)\right|^{p}\right. \\
+ & \left.\left|h_{n+1}\left(x_{n}(s), x_{n}(q s), u\right)-h_{n}(s, u)\right|^{p}\right) \pi(d u) d s .
\end{aligned}
$$


For $t_{0} \leq t \leq \tau_{n}$, we have

$$
\begin{array}{r}
f_{n+1}\left(t, x_{n}(t), x_{n}(q t)\right)=f_{n}(t)=f\left(t, x_{n}(t), x_{n}(q t)\right), \\
g_{n+1}\left(t, x_{n}(t), x_{n}(q t)\right)=g_{n}(t)=g\left(t, x_{n}(t), x_{n}(q t)\right), \\
h_{n+1}\left(x_{n}(t), x_{n}(q t), u\right)=h_{n}(t, u)=h\left(x_{n}(t), x_{n}(q t), u\right) .
\end{array}
$$

By (33), we get from (32),

$$
\begin{aligned}
& E \sup _{t_{0} \leq s \leq t}\left|r_{n}(s)\right|^{p} \\
\leq & \left(6\left(T-t_{0}\right)\right)^{p-1} E \int_{t_{0}}^{t}\left|f_{n+1}(s)-f_{n+1}\left(s, x_{n}(s), x_{n}(q s)\right)\right|^{p} d s \\
& +6^{p-1} c_{p}\left(T-t_{0}\right)^{\frac{p}{2}-1} E \int_{t_{0}}^{t}\left|g_{n+1}(s)-g_{n+1}\left(s, x_{n}(s), x_{n}(q s)\right)\right|^{p} d s \\
& +\left(6\left(T-t_{0}\right)\right)^{p-1}[2 \pi(U)]^{\frac{p}{2}} E \int_{t_{0}}^{t}\left[\int_{U} \mid h_{n+1}(s, u)\right. \\
& \left.-\left.h_{n+1}\left(x_{n}(s), x_{n}(q s), u\right)\right|^{2} \pi(d u)\right]^{\frac{p}{2}} d s \\
& +6^{p-1} D_{p} 2^{\frac{p}{2}} E\left[\int_{t_{0}}^{t} \int_{U}\left|h_{n+1}(s, u)-h_{n+1}\left(x_{n}(s), x_{n}(q s), u\right)\right|^{2} \pi(d u) d s\right]^{\frac{p}{2}} \\
& +12^{p-1} D_{p} E \int_{t_{0}}^{t} \int_{U}\left|h_{n+1}(s, u)-h_{n+1}\left(x_{n}(s), x_{n}(q s), u\right)\right|^{p} \pi(d u) d s .
\end{aligned}
$$

By the local Lipschitz condition (H3) and the basic inequality $|a+b|^{p} \leq$ $2^{p-1}\left(|a|^{p}+|b|^{p}\right)$, we have

$$
\begin{aligned}
E \sup _{t_{0} \leq s \leq t}\left|r_{n}(s)\right|^{p} & \left.\leq\left. C E \int_{t_{0}}^{t}\left[\left|r_{n}(s)\right|^{p}+\mid r_{n}(q s)\right)\right|^{p}\right] d s \\
& \leq 2 C \int_{t_{0}}^{t} E \sup _{t_{0} \leq \sigma \leq s}\left|r_{n}(\sigma)\right|^{p} d s,
\end{aligned}
$$

where $C=\left(12\left(T-t_{0}\right)\right)^{p-1}\left(k_{n} \pi(d u)\right)^{\frac{p}{2}}+12^{p-1} D_{p}\left[\left(k_{n}\left(T-t_{0}\right)\right)^{\frac{p}{2}}+k_{n}\right]$. Thus, the Gronwall inequality implies that

$$
E \sup _{t_{0} \leq s \leq t}\left|x_{n+1}(s)-x_{n}(s)\right|^{p}=0, \quad t_{0} \leq t \leq \tau_{n} .
$$

Therefore we obtain that

$$
x_{n+1}(t)=x_{n}(t), \quad \text { for } \quad t \in\left[t_{0}, \tau_{n}\right] .
$$


It then deduced that $\tau_{n}$ is increasing, that is as $n \rightarrow \infty, \tau_{n} \uparrow T$ a.s. By the linear growth condition (H2), for almost all $\omega \in \Omega$, there exists an integer $n_{0}=n_{0}(\omega)$ such that $\tau_{n}=T$ as $n \geq n_{0}$. Now define $x(t)$ by $x(t)=x_{n_{0}}(t)$ for $t \in\left[t_{0}, T\right]$. Next to verify that $x(t)$ is a solution of equation (3). By (34), $x\left(t \wedge \tau_{n}\right)=x_{n}\left(t \wedge \tau_{n}\right)$, and by $(27)$, it follows that

$$
\begin{aligned}
x\left(t \wedge \tau_{n}\right)= & \varphi(t)+\int_{t_{0}}^{t \wedge \tau_{n}} f_{n}(s) d s+\int_{t_{0}}^{t \wedge \tau_{n}} g_{n}(s) d w_{s} \\
& +\int_{t_{0}}^{t \wedge \tau_{n}} \int_{U} h_{n}(s, u) N_{\bar{p}}(d s, d u) \\
= & \varphi(t)+\int_{t_{0}}^{t \wedge \tau_{n}} f(s, x(s), x(q s)) d s+\int_{t_{0}}^{t \wedge \tau_{n}} g(s, x(s), x(q s)) d w_{s} \\
& +\int_{0}^{t \wedge \tau_{n}} \int_{U} h(x(s), x(q s), u) N_{\bar{p}}(d s, d u) .
\end{aligned}
$$

Letting $n \rightarrow \infty$, then yields

$$
\begin{aligned}
x(t \wedge T)= & \varphi(t)+\int_{t_{0}}^{t \wedge T} f(s, x(s), x(q s)) d s+\int_{t_{0}}^{t \wedge T} g(s, x(s), x(q s)) d w_{s} \\
& +\int_{t_{0}}^{t \wedge T} \int_{U} h(x(s), x(q s), u) N_{\bar{p}}(d s, d u) .
\end{aligned}
$$

That is

$$
\begin{aligned}
x(t)= & \varphi(t)+\int_{t_{0}}^{t} f(s, x(s), x(q s)) d s+\int_{t_{0}}^{t} g(s, x(s), x(q s)) d w_{s} \\
& +\int_{t_{0}}^{t} \int_{U} h(x(s), x(q s), u) N_{\bar{p}}(d s, d u) .
\end{aligned}
$$

we can see that $x(t)$ is the solution of equation (3) and $x(t) \in \mathcal{M}^{p}\left(\left[t_{0}, T\right] ; R^{n}\right)$. The existence of solution to equation (3) is complete. By stopping our process, uniqueness is obtained. The proof is complete.

\section{Asymptotic estimations for solutions}

In this section, we will study the exponential estimate of solution to equation (3). 
According to the definition of $\tilde{N}_{\bar{p}}(d t, d u):=N_{\bar{p}}(d t, d u)-\pi(d u) d t$, we can rewrite equation (2) as the following equation

$$
\begin{aligned}
d x(t)= & F(t, x(t), x(q t), u) d t+g(t, x(t), x(q t)) d w_{t} \\
& +\int_{U} h(x(t), x(q t), u) \tilde{N}_{\bar{p}}(d t, d u)
\end{aligned}
$$

where $F(t, x(t), x(q t), u)=f(t, x(t), x(q t))+\int_{U} h(x(t), x(q t), u) \pi(d u)$.

Let $C^{2}\left(R^{n} \times R_{+}, R_{+}\right)$denote the family of all nonnegative functions $V(x, t)$ on $R^{n} \times R_{+}$which are continuously twice differentiable in $x$. For each $V \in$ $C^{2}\left(R^{n} \times R_{+}, R_{+}\right)$, define an operator $L V$ from $R^{n} \times R^{n} \times R_{+}$to $R$ by

$$
\begin{aligned}
& L V(x, y, t) \equiv V_{t}(x, t)+V_{x}(x, t) F(t, x, y, u) \\
& +\frac{1}{2} \operatorname{trace}\left[g^{\top}(t, x, y) V_{x x}(x, t) g(t, x, y)\right] \\
& +\int_{U}\left[V(x+h(x, y, u), t)-V(x, t)-V_{x}(x, t) h(x, y, u)\right] \pi(d u)
\end{aligned}
$$

where

$$
\begin{gathered}
V_{t}(x, t)=\frac{\partial V(x, t)}{\partial t}, \quad V_{x}(x, t)=\left(\frac{\partial V(x, t)}{\partial x_{1}}, \cdots, \frac{\partial V(x, t}{\partial x_{n}}\right), \\
V_{x x}(x, t)=\left(\frac{\partial^{2} V(x, t)}{\partial x_{i} \partial x_{j}}\right)_{n \times n} .
\end{gathered}
$$

First, we establish the $p$-th exponential estimations of solution to equation (3).

Theorem 4.1 Let $\left\{x(t), t_{0} \leq t \leq T\right\}$ be a solution of equation (3) whose coefficients satisfy conditions $(\mathrm{H} 1)$, (H2). Then, for any $p \geq 2$, there exist two constants $M_{1}, M_{2}$ such that

$$
E \sup _{t_{0} \leq s \leq t}|x(s)|^{p} \leq\left[2\left(1+E\|\varphi\|^{p}\right)+M_{1}\left(T-t_{0}\right)\right] e^{M_{2}\left(t-t_{0}\right)} .
$$

Proof: Let $V(t, x(t))=1+|x(t)|^{p}$, then $V_{t}(t, x(t))=0$. Applying the It $\widehat{o}$ formula to $V(t, x(t))$, we obtain

$$
V(t, x(t))=V\left(t_{0}, x\left(t_{0}\right)\right)+\int_{t_{0}}^{t} L V(x(s), x(q s), s) d s
$$




$$
\begin{aligned}
& +\int_{t_{0}}^{t} V_{x}(s, x(s)) g(s, x(s), x(q s)) d w_{s} \\
& +\int_{t_{0}}^{t} \int_{U}[V(x(s)+h(x(s), x(q s), u), s)-V(x(s), s)] \tilde{N}_{\bar{p}}(d s, d u) .
\end{aligned}
$$

Let $f_{t}=f(t, x(t), x(q t)), g_{t}=g(t, x(t), x(q t)), h_{t, u}=h(x(t), x(q t), u), F_{t, u}=$ $f_{t}+\int_{U} h_{t, u} \pi(d u)$. By (38), we obtain that

$$
\begin{aligned}
& 1+|x(t)|^{p}=1+\left|x\left(t_{0}\right)\right|^{p}+p \int_{t_{0}}^{t}|x(s)|^{p-2}\left(x(s), F_{s, u}\right) d s \\
& +\frac{1}{2} p \int_{t_{0}}^{t}|x(s)|^{p-2}\left|g_{s}\right|^{2} d s+\frac{p(p-2)}{2} \int_{t_{0}}^{t}|x(s)|^{p-4}\left|\left(x(s), g_{s}\right)\right|^{2} d s \\
& +p \int_{t_{0}}^{t}|x(s)|^{p-2}\left(x(s), g_{s} d w_{s}\right) \\
& +\int_{t_{0}}^{t} \int_{U}\left\{\left(1+\left|x(s)+h_{s, u}\right|^{p}\right)-\left(1+|x(s)|^{p}\right)-p|x(s)|^{p-2}\left(x(s), h_{s, u}\right)\right\} \pi(d u) d s \\
& +\int_{t_{0}}^{t} \int_{U}\left\{\left(1+\left|x(s)+h_{s, u}\right|^{p}\right)-\left(1+|x(s)|^{p}\right)\right\} \tilde{N}_{\bar{p}}(d s, d u) .
\end{aligned}
$$

Taking expectation on $1+|x(t)|^{p}$ and using the basic inequality $2 a b \leq a^{2}+b^{2}$, we have

$$
\begin{aligned}
& E \sup _{t_{0} \leq s \leq t}\left(1+|x(s)|^{p}\right) \\
\leq & 1+E\|\varphi\|^{p}+\frac{p}{2} E \int_{t_{0}}^{t}|x(s)|^{p} d s+\frac{p}{2} E \int_{t_{0}}^{t}|x(s)|^{p-2}\left|F_{s, u}\right|^{2} d s \\
& +\frac{p(p-1)}{2} E \int_{t_{0}}^{t}|x(s)|^{p-2}\left|g_{s}\right|^{2} d s \\
& +p E \sup _{t_{0} \leq s \leq t} \int_{t_{0}}^{s}|x(\sigma)|^{p-2}\left(x(\sigma), g_{\sigma} d w_{\sigma}\right) \\
& +p E \sup _{t_{0} \leq s \leq t} \int_{t_{0}}^{s} \int_{U}|x(\sigma)|^{p-2}\left(x(\sigma), h_{\sigma, u}\right) \tilde{N}_{\bar{p}}(d \sigma, d u) \\
& +E \sup _{t_{0} \leq s \leq t} \int_{t_{0}}^{s} \int_{U}\left\{\left|x(\sigma)+h_{\sigma, u}\right|^{p}-|x(\sigma)|^{p}\right. \\
& \left.-p|x(\sigma)|^{p-2}\left(x(\sigma), h_{\sigma, u}\right)\right\} N_{\bar{p}}(d \sigma, d u) \\
\leq & 1+E\|\varphi\|^{p}+\frac{p}{2} E \int_{t_{0}}^{t}|x(s)|^{p} d s+\mathcal{J}_{1}+\mathcal{J}_{2}+\mathcal{J}_{3}+\mathcal{J}_{4}+\mathcal{J}_{5} .
\end{aligned}
$$


Let us estimate $\mathcal{J}_{1}$. By the Young inequality, we have

$$
\mathcal{J}_{1} \leq \frac{p}{2}\left[\frac{p-2}{p} E \int_{t_{0}}^{t}|x(s)|^{p} d s+\frac{2}{p} E \int_{t_{0}}^{t}\left|F_{s, u}\right|^{p} d s .\right.
$$

Using the basic inequality and (H2), we obtain

$$
\left.E \int_{t_{0}}^{t}\left|F_{s, u}\right|^{p} d s \leq c_{10}\left(T-t_{0}\right)+2 c_{10} \int_{t_{0}}^{t} E \sup _{t_{0} \leq \sigma \leq s}|x(\sigma)|^{p} d s\right] .
$$

where $c_{10}=2^{p-1} 3^{\frac{p}{2}-1}\left[L_{1}^{\frac{p}{2}}+\left(L_{2} \pi(U)\right)^{\frac{p}{2}}\right]$. Then, we get

$$
\mathcal{J}_{1} \leq c_{10}\left(T-t_{0}\right)+\left[\frac{p-2}{2}+2 c_{10}\right] \int_{t_{0}}^{t} E \sup _{0 \leq \sigma \leq s}|x(\sigma)|^{p} d s .
$$

Similarly, we obtain that

$$
\mathcal{J}_{2} \leq c_{11}\left(T-t_{0}\right)+\left[\frac{p-2}{2}+2 c_{11}\right] \int_{t_{0}}^{t} E \sup _{0 \leq \sigma \leq s}|x(\sigma)|^{p} d s
$$

where $c_{11}=(p-1) L_{1}^{\frac{p}{2}} 3^{\frac{p}{2}-1}$. Let us estimate $\mathcal{J}_{5}$. Since $N_{\bar{p}}(d t, d u)=\tilde{N}_{\bar{p}}(d t, d u)+$ $\pi(d u) d t$ and $\tilde{N}_{\bar{p}}(d t, d u)$ is a martingale, it follows that

$$
\mathcal{J}_{5} \leq E \int_{t_{0}}^{t} \int_{U}\left\{\left|x(s)+h_{s, u}\right|^{p}-|x(s)|^{p}-p|x(s)|^{p-2}\left(x(s), h_{s, u}\right)\right\} \pi(d u) d s .
$$

We note that it has the form

$$
E \int_{t_{0}}^{t} \int_{U}\left\{f\left(x(s)+h_{s, u}\right)-f(x(s))-f^{\prime}(x(s)) h_{s, u}\right\} \pi(d u) d s,
$$

where $f(x)=|x|^{p}$. Using the Taylor formula, there exists a positive constant $M_{p}(p \geq 2)$ such that

$$
\begin{aligned}
& f\left(x(s)+h_{s, u}\right)-f(x(s))-f^{\prime}(x(s)) h_{s, u} \\
= & \left|x(s)+h_{s, u}\right|^{p}-|x(s)|^{p}-p|x(s)|^{p-2}\left(x(s), h_{s, u}\right) \\
\leq & M_{p}\left[\left|x(s)+h_{s, u}\right|^{p-2}\left|h_{s, u}\right|^{2}+\left|h_{s, u}\right|^{p}\right] .
\end{aligned}
$$


Thus, using the basic inequality $|a+b|^{p-2} \leq 2^{p-3}\left(|a|^{p-2}+|b|^{p-2}\right)$, the Young inequality and (H2), we obtain that

$$
\begin{aligned}
\mathcal{J}_{5} \leq & M_{p} E \int_{t_{0}}^{t} \int_{U}\left[\left|x(s)+h_{s, u}\right|^{p-2}\left|h_{s, u}\right|^{2}+\left|h_{s, u}\right|^{p}\right] \pi(d u) d s \\
\leq & M_{p} 2^{p-3} \frac{p-2}{p} E \int_{t_{0}}^{t} \int_{U}|x(s)|^{p} \pi(d u) d s \\
& +M_{p}\left[2^{p-3}\left(1+\frac{2}{p}\right)+1\right] E \int_{t_{0}}^{t} \int_{U}\left|h_{s, u}\right|^{p} \pi(d u) d s \\
\leq & M_{p} 2^{p-3} \frac{p-2}{p} \pi(U) E \int_{t_{0}}^{t}|x(s)|^{p} d s \\
& +M_{p}\left[2^{p-3}\left(1+\frac{2}{p}\right)+1\right] L_{2} E \int_{t_{0}}^{t}\left(1+|x(s)|^{p}+|x(q s)|^{p}\right) d s \\
\leq & c_{12}\left(T-t_{0}\right)+c_{13} \int_{t_{0}}^{t} E \sup _{t_{0} \leq \sigma \leq s}|x(\sigma)|^{p} d s .
\end{aligned}
$$

where $c_{12}=M_{p}\left[2^{p-3}\left(1+\frac{2}{p}\right)+1\right] L_{2}$ and $c_{13}=M_{p} 2^{p-3} \frac{p-2}{p} \pi(U)+2 M_{p}\left[2^{p-3}(1+\right.$ $\left.\left.\frac{2}{p}\right)+1\right] L_{2}$. For the estimation of $\mathcal{J}_{3}$, by the Burkholder-Davis-Gundy inequality, we have

$$
\begin{aligned}
\mathcal{J}_{3} & \leq 3 p E\left[\int_{t_{0}}^{t}|x(s)|^{2 p-2}\left|g_{s}\right|^{2} d s\right]^{\frac{1}{2}} \\
& \leq 3 p E\left[\sup _{t_{0} \leq s \leq t}|x(s)|^{p}\left(\int_{t_{0}}^{t}|x(s)|^{p-2}\left|g_{s}\right|^{2} d s\right)\right]^{\frac{1}{2}}
\end{aligned}
$$

For any $\varepsilon_{1}>0$, the Young inequality implies that

$$
\begin{aligned}
\mathcal{J}_{3} \leq & 3 p\left[\varepsilon_{1} E \sup _{t_{0} \leq s \leq t}|x(s)|^{p}\right]^{\frac{1}{2}}\left[\frac{1}{\varepsilon_{1}} E\left(\int_{t_{0}}^{t}|x(s)|^{p-2}\left|g_{s}\right|^{2} d s\right)\right]^{\frac{1}{2}} \\
\leq & \frac{3 p \varepsilon_{1}}{2} E \sup _{t_{0} \leq s \leq t}|x(s)|^{p}+\frac{3 p}{2 \varepsilon_{1}} E \int_{t_{0}}^{t}|x(s)|^{p-2}\left|g_{s}\right|^{2} d s \\
\leq & \frac{3 p \varepsilon_{1}}{2} E \sup _{t_{0} \leq s \leq t}|x(s)|^{p}+\frac{\left(T-t_{0}\right)}{\varepsilon_{1}}\left(3 L_{1}\right)^{\frac{p}{2}} \\
& +\left[\frac{3(p-2)}{2 \varepsilon_{1}}+\frac{2}{\varepsilon_{1}}\left(3 L_{1}\right)^{\frac{p}{2}}\right] \int_{t_{0}}^{t} E \sup _{t_{0} \leq \sigma \leq s}|x(\sigma)|^{p} d s .
\end{aligned}
$$


Let $\varepsilon_{1}=\frac{1}{6 p}$, it follows from (47) that

$$
\begin{aligned}
\mathcal{J}_{3} \leq & \frac{1}{4} E \sup _{t_{0} \leq s \leq t}|x(s)|^{p}+6 p\left(3 L_{1}\right)^{\frac{p}{2}}\left(T-t_{0}\right) \\
& +c_{14} \int_{t_{0}}^{t} E \sup _{t_{0} \leq \sigma \leq s}|x(\sigma)|^{p} d s
\end{aligned}
$$

where $c_{14}=9 p(p-2)+12 p\left(3 L_{1}\right)^{\frac{p}{2}}$. Finally, we will give the estimate of $\mathcal{J}_{4}$. By applying the Burkholder-Davis-Gundy inequality again, there exists a positive constant $\tilde{c}_{p}$ such that

$$
\begin{aligned}
\mathcal{J}_{4} & \leq p \tilde{c}_{p} E\left[\int_{t_{0}}^{t} \int_{U}|x(s)|^{2 p-2}\left|h_{s, u}\right|^{2} \pi(d u) d s\right]^{\frac{1}{2}} \\
& \leq p \tilde{c}_{p} E\left[\sup _{t_{0} \leq s \leq t}|x(s)|^{p}\left(\int_{t_{0}}^{t} \int_{U}|x(s)|^{p-2}\left|h_{s, u}\right|^{2} \pi(d u) d s\right)\right]^{\frac{1}{2}} .
\end{aligned}
$$

Further, using the Young inequality again, we have for any $\varepsilon_{2}>0$

$$
\begin{aligned}
\mathcal{J}_{4} \leq & p \tilde{c}_{p}\left[\varepsilon_{2} E \sup _{t_{0} \leq s \leq t}|x(s)|^{p}\right]^{\frac{1}{2}}\left[\frac{1}{\varepsilon_{2}} E\left(\int_{t_{0}}^{t} \int_{U}|x(s)|^{p-2}\left|h_{s, u}\right|^{2} \pi(d u) d s\right)\right]^{\frac{1}{2}} \\
\leq & \frac{p \tilde{c}_{p} \varepsilon_{2}}{2} E \sup _{t_{0} \leq s \leq t}|x(s)|^{p}+\frac{p \tilde{c}_{p}}{2 \varepsilon_{2}} E \int_{t_{0}}^{t} \int_{U}|x(s)|^{p-2}\left|h_{s, u}\right|^{2} \pi(d u) d s \\
\leq & \frac{p \tilde{c}_{p} \varepsilon_{2}}{2} E \sup _{t_{0} \leq s \leq t}|x(s)|^{p}+\frac{p \tilde{c}_{p}}{2 \varepsilon_{2}}\left[\frac{p-2}{p} E \int_{t_{0}}^{t} \int_{U}|x(s)|^{p} \pi(d u) d s\right. \\
& \left.+\frac{2}{p} E \int_{0}^{t} \int_{U}\left|h_{s, u}\right|^{p} \pi(d u) d s\right] \\
\leq & \frac{p \tilde{c}_{p} \varepsilon_{2}}{2} E \sup _{t_{0} \leq s \leq t}|x(s)|^{p}+\frac{\tilde{c}_{p}}{\varepsilon_{2}} L_{2}\left(T-t_{0}\right) \\
& +\left[\frac{\tilde{c}_{p}(p-2) \pi(U)}{2 \varepsilon_{2}}+\frac{2 \tilde{c}_{p}}{\varepsilon_{2}} L_{2}\right] \int_{t_{0}}^{t} E \sup _{t_{0} \leq \sigma \leq s}|x(\sigma)|^{p} d s .
\end{aligned}
$$

Let $\varepsilon_{2}=\frac{1}{2 p \tilde{c}_{p}}$, we obtain that

$$
\mathcal{J}_{4} \leq \frac{1}{4} E \sup _{t_{0} \leq s \leq t}|x(s)|^{p}+2 p \tilde{c}_{p}^{2} L_{2}\left(T-t_{0}\right)+c_{15} \int_{t_{0}}^{t} E \sup _{t_{0} \leq \sigma \leq s}|x(\sigma)|^{p} d s,(50)
$$


where $c_{15}=p(p-2) \tilde{c}_{p}^{2} \pi(U)+2 p \tilde{c}_{p}^{2} L_{2}$. Combing $\mathcal{J}_{1}-\mathcal{J}_{5}$ and (39) together, we have

$$
\begin{aligned}
E \sup _{t_{0} \leq s \leq t}\left(1+|x(s)|^{p}\right) \leq & 2\left(1+E\|\varphi\|^{p}\right)+M_{1}\left(T-t_{0}\right) \\
& +M_{2} \int_{t_{0}}^{t} E \sup _{0 \leq \sigma \leq s}\left(1+|x(\sigma)|^{p}\right) d s
\end{aligned}
$$

where

$$
\begin{aligned}
& M_{1}=2\left[c_{10}+c_{11}+c_{12}+6 p\left(3 L_{1}\right)^{\frac{p}{2}}+2 p \tilde{c}_{p}^{2} L_{2}\right], \\
& M_{2}=2\left(\frac{3 p}{2}-2+2 c_{10}+2 c_{11}+c_{13}+c_{14}+c_{15}\right) .
\end{aligned}
$$

Therefore, we apply the Gronwall inequality to get

$$
E \sup _{t_{0} \leq s \leq t}\left(1+|x(s)|^{p}\right) \leq\left[2\left(1+E\|\varphi\|^{p}\right)+M_{1}\left(T-t_{0}\right)\right] e^{M_{2}\left(t-t_{0}\right)} .
$$

This completes the proof.

The next result shows that exponential estimations implies almost surely asymptotic estimations, and we give an upper bound for the sample Lyapunov exponent.

Theorem 4.2 Under conditions (H1), (H2), we have

$$
\limsup _{t \rightarrow \infty} \frac{1}{t} \log |x(t)| \leq 1+78 L_{1}+\left(2+4 \pi(U)+8 \tilde{c}_{2}^{2}\right) L_{2}, \quad \text { a.s. }
$$

That is, the sample Lyapunov exponent of the solution should not be greater than $1+78 L_{1}+\left(2+4 \pi(U)+8 \tilde{c}_{2}^{2}\right) L_{2}$.

Proof: For each $n=1,2, \ldots$, it follows from Theorem 4.1 (taking $p=2$ ) that

$$
E\left(\sup _{t_{0}+n-1 \leq t \leq t_{0}+n}|x(t)|^{2}\right) \leq \beta e^{\gamma n}
$$

where $\beta=2\left(1+E\|\varphi\|^{2}\right)+2\left[39 L_{1}+\left(1+2 \pi(U)+4 \tilde{c}_{2}^{2}\right) L_{2}\right]\left(T-t_{0}\right)$ and $\gamma=$ $2\left[1+78 L_{1}+\left(2+4 \pi(U)+8 \tilde{c}_{2}^{2}\right) L_{2}\right]$. Hence, for any $\varepsilon>0$, by the chebysher inequality, it follows that

$$
P\left\{\omega: \sup _{t_{0}+n-1 \leq t \leq t_{0}+n}|x(t)|^{2}>e^{(\gamma+\epsilon) n}\right\} \leq \beta e^{-\epsilon n} .
$$


Since $\Sigma_{n=0}^{\infty} \beta e^{-\varepsilon n}<\infty$, by Borel-Cantelli lemma, we deduce that, there exists a integer $n_{0}$ such that

$$
\sup _{t_{0}+n-1 \leq t \leq t_{0}+n}|x(t)|^{2} \leq e^{(\gamma+\varepsilon) n} \quad \text { a.s } n \geq n_{0} .
$$

Thus, for almost all $\omega \in \Omega$, if $t_{0}+n-1 \leq t \leq t_{0}+n$ and $n \geq n_{0}$, then

$$
\frac{1}{t} \log |x(t)|=\frac{1}{2 t} \log \left(|x(t)|^{2}\right) \leq \frac{(\gamma+\varepsilon) n}{2\left(t_{0}+n-1\right)} \text {. }
$$

Taking lim sup in (52) leads to almost surely exponential estimate, that is,

$$
\limsup _{t \rightarrow \infty} \frac{1}{t} \log |x(t)| \leq \frac{\gamma+\varepsilon}{2}=1+78 L_{1}+\left(2+4 \pi(U)+8 \tilde{c}_{2}^{2}\right) L_{2}, \quad \text { a.s. }
$$

Required assertion (51) follows because $\varepsilon>0$ is arbitrary.

\section{Acknowledgements}

The authors would like to thank the Royal Society of Edinburgh, the National Natural Science Foundation of China under NSFC grant (No.11401261), Qing Lan Project of Jiangsu Province (2012), the NSF of Higher Education Institutions of Jiangsu Province (13KJB110005), the grant of Jiangsu Second Normal University (JSNU-ZY-02) and the Jiangsu Government Overseas Study Scholarship for their financial support.

\section{References}

[1] L. Fox, D. F. Mayers, J. R. Ockendon and A. B. Tayler, On a functional differential equation, J. Inst. Math. Appl, 8 (1971), 271-307.

[2] J. R. Ockendon and A. B. Tayler, The dynamics of a current collection system for an electric locomotive, Proc. Roy. Soc. London Ser. A, 322 (1971), 447-468.

[3] J. Carr and J. Dyson, The functional differential equation $y^{\prime}(x)=a y(\lambda x)+$ by $(x)$, Proc. Roy. Soc. Edinburgh Sect. A, 74 (1974), 165-174.

[4] T. Kato and J. B. Mcleod, The functional-differential equation $y^{\prime}(x)=$ $a y(\lambda x)+b y(x)$, Bull. Amer. Math. Soc, 77 (1971), 891-937.

[5] Y. Liu, Numerical investigation of the pantograph equation, Appl. Numer. Math, 24 (1997), 309-317. 
[6] H. Brunner, On the discretization of differential and Volterra integral equations with variable delay, BIT Numerical Mathematics, 37 (1997), 1-12.

[7] J. K. Hale, Introduction to Functional Differential Equations, SpringerVerlag, New York, 1993.

[8] A. Iserles, On the generalized pantograph functional-differential equation, European J. Appl. Math, 4 (1993), 1-38.

[9] M. Z. Liu, Z. W. Yang, G. D. Hu, Asymptotical stability of the numerical methods with the constant stepsize for the pantograph equation, Numerical Mathematics, 45 (2005), 743-759.

[10] S. E. A. Mohammed, Stochastic Functional Differential Equations, Longman Scientific and Technical, 1984.

[11] X. Mao, Stochastic Differential Equations and Applications, 2nd ed., Woodhead Publishing Limited, 2011.

[12] X. Mao, Robustness of exponential stability of stochastic differential delay equations, IEEE Transactions on Automatic Control, 41 (1996), 442-447.

[13] X. Mao and M. J. Rassias, Khasminskii-type theorems for stochastic differential delay equations, J. Sto. Anal. Appl, 23 (2005), 1045-1069.

[14] X. Mao, Numerical solutions of stochastic differential delay equations under the generalized Khasminskii-type conditions, Appl. Math. Comput, 217 (2011), 5512-5524.

[15] U. Kuchler and E. Platen, Strong discrete time approximation of stochastic differential equations with time delay, Math. Comput. Simulation, 54 (2000), 189-205.

[16] E. Buckwar, Introduction to the numerical analysis of stochastic delay differential equations, J. Comput.Appl.Math, 125(2000), 297-307.

[17] E. Buckwar, T. Shardlow, Weak approximation of stochastic differential delay equations, IMA J Numer Anal, 25 (2005),57-86.

[18] Y. Hu, S. E. A. Mohammed, F. Yan, Discrete-time approximation of stochastic delay equations: the Milstein scheme, Ann.Probab, 32 (2004), 265-314.

[19] F. Wu, X. Mao, Numerical solutions of neutral stochastic functional differential equations, SIAM J. Numer. Anal, 46(2008),1821-1841. 
[20] F. Wu, X. Mao and L. Szpruch, Almost sure exponential stability of numerical solutions for SDDEs, Numerische Mathematik, 115 (2010), 681-697.

[21] J. Appleby and E. Buckwar, Sufficient conditions for polynomial asymptotic behavior of the stochastic pantograph equation, available at http://www.dcu.ie./maths/research/ preprint.shtml. 2003.

[22] Z. Fan, M. Liu, W. Cao, Existence and uniqueness of the solutions and convergence of semi-implicit Euler methods for stochastic pantograph equations, J. Math. Anal. Appl, 325 (2007), 1142-1159.

[23] Z. Fan, M. Song, The $\alpha$ th moment stability for the stochastic pantograph equation, J. Comput. Appl. Math, 233 (2009), 109-120.

[24] C. T. H. Baker,E.Buckwar, Continuous $\theta$-methods for the stochastic pantograph equation, Elect Trans Numer Anal, 11 (2000), 131-151.

[25] R. Li, M. Liu, W. Pang, Convergence of numerical solutions to stochastic pantograph equations with Markovian switching, Appl. Math. Comput, 215(2009),414-422.

[26] Y. Xiao, H. Y. Zhang, Convergence and stability of numerical methods with variable step size for stochastic pantograph differential equations, I. J. Comput. Math, 88(2011), 2955-2968.

[27] Y. Xiao, M. Song, M. Liu, Convergence and stability of the semi-implicit Euler method with variable step size for a linear stochastic pantograph differential equation, Int. J. Numer. Anal. Model, 8 (2011), 214-225.

[28] Z. H. Yu, Almost Surely Asymptotic Stability of Exact and Numerical Solutions for Neutral Stochastic Pantograph Equations, Abstract and Applied Analysis, Volume 2011 (2011). http://dx.doi.org/10.1155/2011/143079.

[29] M. Marija, J. Miljana, A Taylor polynomial approach in approximations of solution to pantograph stochastic differential equations with Markovian switching, Math. Comput. Modelling, 53 (2011), 280-293.

[30] R. C. Merton, Option pricing when underlying stock returns are discontinuous, J. Financ. Econ, 2 (1976), 125-144.

[31] P. Jorion, On jump processes in the foreign exchange and stock markets, Rev. Financ. Studies, 1 (1988), 427-445. 
[32] R. C. Merton, Continuous-Time Finance, Basil Blackwell, Cambridge, MA, 1990.

[33] Y. Maghsoodi, Mean square efficient numerical solution of jump-diffusion stochastic differential equations, Indian J. Statist, 58 (1996), 25-47.

[34] A. Gardon, The order of approximations for solutions of Ito-type stochastic differential equations with jumps, Stochastic Anal. Appl, 22 (2004), 679-699.

[35] D. Higham, P. Kloeden, Numerical methods for nonlinear stochastic differential equations with jumps, Numer. Math, 110 (2005), 101-119.

[36] N. Bruti-Liberati and E. Platen, Approximation of jump diffusions in finance and economics, Computational Economics, 29 (2007), 283-312.

[37] N. Ikeda, S. Watanabe, Stochastic Differential Equations and Diffusion Processes, North Holland Publishing Company, Amsterdam, Oxford, New York, 1989.

[38] D. Applebaum, Levy Processes and Stochastic Calculus, 2nd ed., Cambridge University Press, 2011.

[39] S. T. Rong, Theory of Stochastic Differential Equations with Jumps and Applications, Springer, Berlin, 2005.

[40] R. Bass, Stochastic differential equations with jumps, Probability Surveys, 1 (2004), 1-19.

[41] S. Albeverio, Z. Brzezniak, J. Wu, Existence of global solutions and invariant measures for stochastic differential equations driven by Poisson type noise with non-Lipschitz coefficients, J. Math. Anal. Appl, 371 (2010), 309-322.

[42] B. Oksendal, A. Sulem, Applied stochastic control of jump diffusions, Springer, Berlin, 2005.

[43] C. W. Li, Z. Dong, S. T. Rong, Almost sure stability of linear stochastic differential equations with jumps, Probability Theory and Related Fields, 123 (2002), 121-155.

[44] D. Applebaum, M. Siakalli, Asymptotic stability of stochastic differential equations driven by Lévy noise, J. Appl. Probab, 46 (2009), 1116-1129.

[45] D. Applebaum, M. Siakalli, Stochastic stabilization of dynamical systems using Lévy noise, Stoch. Dyn, 10 (2010), 509-527. 
[46] Q. Zhu, Asymptotic stability in the pth moment for stochastic differential equations with Lévy noise, J. Math. Anal. Appl, 416 (2014), 126-142.

[47] H. Kunita, Stochastic differential equations based on Lévy processes and stochastic flows of diffeomorphisms, in Real and Stochastic Analysis, New Perspectives, ed. M. M. Rao, Birkhauser Boston Basel Berlin, (2004), 305-75.

[48] P. Billingsley, Convergence of probability measures, JohnWiley and Sons, Inc., New York-London- Sydney, 1968. 